\title{
Macro-diseminación de la cultura maker: promoviendo competencias del siglo XXI a través de un Ideatón
}

\author{
Macro-dissemination of maker culture: \\ fostering 21 st century competencies through an Ideaton
}

\author{
Jorge Sanabria- $Z^{1}$ \\ Tecnológico de Monterrey, Guadalajara, México \\ jorge.sanabria@tec.mx \\ Ann-Louise Davidson \\ Concordia University, Montreal, Canadá \\ ann-louise.davidson@concordia.ca \\ Margarida Romero \\ Université Côte d'Azur, Nice, France \\ margarida.romero@univ-cotedazur.fr \\ Teresa Quintana \\ Secretaría de Innovación, Ciencia y Tecnología, Guadalajara, México \\ teresa.quintana@jalisco.gob.mx
}

\begin{abstract}
Resumen
Con el objetivo de promover la cultura de innovación y el emprendimiento a través de la ideación dirigida, se llevó a cabo una competición co-tecno-creativa para 1000 estudiantes de bachillerato, durante el Festival de Innovación Epicentro 2018 en Jalisco, México. Los objetivos de la competición estuvieron alineados a los Objetivos de Desarrollo Sostenible de la Organización de las Naciones Unidas, cuya metodología se centró en evocar soluciones prácticas e innovadoras a problemáticas locales de ocho sectores estratégicos del Estado. La actividad de prototipado se caracterizó por la inclusión de microcontroladores programables, sensores y motores, que permiten el desarrollo de competencias del siglo XXI tales como creatividad, colaboración, pensamiento crítico, pensamiento computacional y resolución de problemas. El seguimiento a los participantes se estableció a un año utilizando una plataforma digital. Se presentan el esquema logístico realizado en colaboración entre la Secretaría de Innovación, Ciencia y Tecnología (SICyT) y la Universidad de Guadalajara, así como el protocolo de investigación planteado entre tres universidades de México, Canadá y Francia.
\end{abstract}

Palabras clave: Educación maker, competencias del siglo 21; aprendizaje colaborativo; creatividad; resolución de problemas; innovación abierta.

\begin{abstract}
With the aim of fostering innovation culture and entrepreneurship through directed ideation, a co-techno-creative competition was held for 1,000 senior high school students, as part of the 2018 Epicentro Innovation Festival in Jalisco, Mexico. The
\end{abstract}

\footnotetext{
${ }^{1}$ Durante la investigación llevada a cabo para este artículo, el autor principal estaba adscrito a la Universidad de Guadalajara. Actualmente está adscrito al Tecnológico de Monterrey, Campus Guadalajara.
} 
competition objectives were aligned with the United Nations' Sustainable Development Goals, whose methodology was based on evoking practical and innovative solutions to the local problems of the eight strategic sectors of the state. The prototyping activity was characterized by the inclusion of programmable microcontrollers, sensors, and actuators, which fosters the development of 21st century competencies such as creativity, collaboration, critical thinking, computational thinking, and problem solving. The participant follow-up, using a digital platform, was set for a year. The logistical scheme developed by the Ministry of Innovation, Science and Technology (SICyT) in collaboration with the Universidad de Guadalajara, and the research design jointly proposed by three universities, in Mexico, Canada, and France, are also presented.

Keywords: Maker education; 21st century competencies; collaborative learning; creativity; problem solving; open innovation.

\section{Introducción}

\subsection{El paradigma de Innovación Abierta 2.0 (OI2)}

La transición de una economía del conocimiento en una economía creativa aplicada requiere de un ecosistema con mecanismos centrados en los emprendedores para apoyar la realización de ideas innovadoras (Florida, 2014). En el caso de México, tradicionalmente los proyectos tecnológicos han sido orquestados por la triada Gobierno + Industria + Academia/Investigación, dejando de lado la inclusión de los usuarios en el proceso, comúnmente percibidos solamente como receptores de la innovación. Esta desarticulación de las hélices afecta a los usuarios como potenciales emprendedores, comprometiendo la visión de sus ideas o proyectos, con el resultado de que, incluso con apoyo financiero, generen prototipos de limitada originalidad y relevancia que jamás llegan a posicionarse en el mercado y, por ende, no logran cruzar el llamado Valle de la Muerte (Markham et al., 2010), una travesía en la que las ideas y prototipos logran sobrevivir o de otra manera "mueren".

El paradigma de Innovación Abierta 2.0 (OI2) ha permitido repensar el lugar de los ciudadanos en este proceso, sumando la hélice "Usuarios/Sociedad" a la triada, generando la cuádruple hélice de la innovación (Carayannis \& Campbell, 2009). El OI2 no solo permite entramar activamente los esfuerzos de las cuatro hélices, sean Gobierno + Industria + Academia/Investigación + Usuarios/Sociedad, sino que prioriza la participación del usuario como arquitecto de sus propias soluciones. Así mismo, promueve el uso colaborativo de tecnologías para la co-tecno-creación, a través de la democratización de los dispositivos de bajo costo, el diseño amigable y la divulgación abierta de información en Internet. Este replanteamiento se observa en la emergencia de una cultura del hacer o cultura maker, un resurgimiento del movimiento Hágalo-ustedmismo (DIY, por sus siglas en inglés) en espacios no formales de aprendizaje, tal como los Fab Lab, espacios creativos abiertos y Hackerspaces, pero también cada vez más visto en las instituciones de educación formal (Domingo-Coscollola, 2018). (ver Figura 1). 


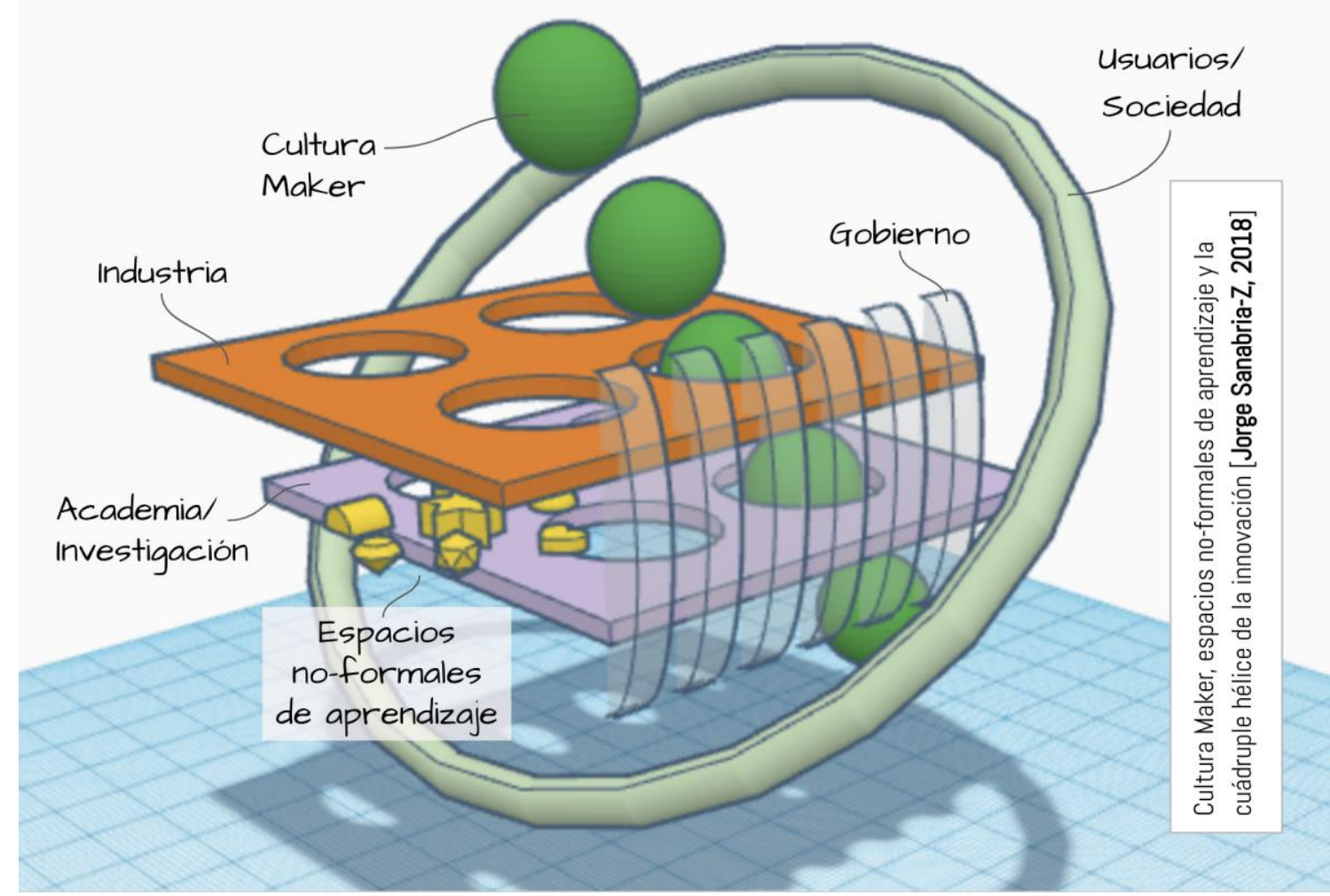

Figura 1. Cultura maker, espacios no formales de aprendizaje y la cuádruple hélice de la innovación (Fuente: Sanabria-Z, 2018).

\subsection{La cultura maker en la educación}

Desde el 2012, la cultura maker, también entendida como un movimiento, ha ganado una gran popularidad en la educación debido a su potencial para traer problemas y desafíos del mundo real al aula y preparar a los estudiantes para las demandas de la sociedad y el mercado laboral previsto (Dougherty, 2012). La cultura maker se permea a través de las cuatro hélices de innovación representadas en la Figura 1, y es particularmente enfatizada en la hélice Academia/Investigación a partir del concepto de educación maker, que se enmarca en el enfoque constructivista del aprendizaje (Fleming, 2015; Papert \& Harel, 1991). Idealmente, en la educación maker los participantes son agentes que crean un artefacto (prototipo, dispositivo tecnológico, etc.) a través del cual aprenden, utilizando herramientas tradicionales (e.g., manuales) y digitales, junto con tecnologías disruptivas, como la impresión 3D, así como materiales tradicionales. En lugar de proyectos individualistas de alto riesgo, como los que tradicionalmente son favorecidos en las escuelas, los makers se involucran en un aprendizaje experiencial autodirigido a través de la resolución persistente de problemas, con tolerancia al riesgo, en comunidades informales interdependientes que abordan problemas socialmente relevantes (Davidson \& Price, 2017). El aprendizaje a través de la creación es interdisciplinario e intergeneracional, y oscila entre contextos formales e informales, lo que puede transformar las prácticas educativas, fomentar la pedagogía progresiva y hacer que el aprendizaje sea más relevante (Cucinelli et al., 2018; Halverson \& Sheridan, 2014).

En los contextos de educación maker, los diseñadores de contextos educativos, proyectos y actividades ofrecen recursos para el desarrollo de las llamadas competencias del siglo XXI, mismos que permiten al participante ubicarse en un contexto de resolución de problemas mediante el uso de la tecnología (Romero \& Gebera, 2012; Sanabria \&

Macro-diseminación de la cultura maker: promoviendo competencias del siglo XXI a través de un Ideatón. Jorge Sanabria-Z, Ann-Louise Davidson, Margarida Romero y Teresa Quintana.

Page 3 of 27 
Romero, 2018; Sanabria-Z \& Romero, 2019). Adicionalmente, a través de actividades tecno-creativas de tipo maker, se promueve el proceso de prototipado de baja y alta definición, impulsando así la calidad y relevancia de nuevas ideas de productos y servicios. Se ha demostrado que contar con una robusta infraestructura de creación de prototipos ayuda a los emprendedores a superar el denominado Valle de la Muerte (Klitsie et al., 2018). De aquí que los esfuerzos por impulsar el desarrollo temprano de ideas y competencias para desarrollarlas utilizando tecnologías disruptivas y de vanguardia, puedan contribuir a la generación y mejora de productos innovadores.

La cultura maker se inscribe en una voluntad por acceder a desarrollar soluciones de bajo coste en un contexto de innovación basada en los ciudadanos. En esta perspectiva, las actividades maker consisten a menudo en hacer pequeños proyectos de bricolaje usando computadoras de código abierto, compactas y asequibles; artículos electrónicos y materiales reciclados para fomentar la sostenibilidad, la equidad, la innovación social, la democratización de la innovación, y la construcción de comunidades (Andersson, 2015). En lugar de centrarse en objetivos de aprendizaje descontextualizados o actividades de aprendizaje individual basadas en puntos a obtener, como es habitual en las escuelas, los proyectos educativos maker desarrollan actividades de equipo basadas en el aprendizaje experiencial autodirigido. Este tipo de aprendizaje puede fomentar la resolución de problemas, la tolerancia a la ambigüedad y la tolerancia al riesgo en contextos de problemas complejos y socialmente relevantes. Los recursos para la educación maker están proliferando en las escuelas (Burker, 2015), las bibliotecas (Haug, 2014) y los centros comunitarios (Sheridan et al., 2014) en varios países.

Sin investigación que estudie no solamente cómo desarrollar el saber-hacer tecnológico, sino también los procesos de pensamiento basados en evidencias de investigación y rastros de reflexión, así como el aprendizaje e iteraciones, los makers corren el riesgo de convertirse en "tecno-fetichistas" que utilizan equipos electrónicos y participan en competiciones técnicas en lugar de centrarse en la innovación social que pueden transmitir las actividades maker (Hertz, 2012); y los espacios maker corren el riesgo de convertirse en meros espacios de alquiler o exhibidores de las obras de los makers, en lugar de promover la educación (Peppler et al., 2016) en comunidades colaborativas que participan en actividades compartidas (Fox, 2014).

Con esto en mente, Davidson et al. (2017) compilaron varios tipos de datos que involucraban a participantes que tomaron parte en actividades formales y no formales dirigidas por makers, tales como talleres maker, improvisaciones (jams), hackatones y retos maker. Los autores identificaron varias actitudes características, tales como tomar la iniciativa, aprender juguetonamente, adaptación auténtica y persistencia; y competencias que incluyen colaboración, co-diseño y planificación conjunta. Además, para tener éxito en las actividades maker, los estudiantes deben desarrollar la capacidad de tolerar errores, lidiar con la incertidumbre y resolver problemas en diferentes niveles de dificultad y complejidad (Davidson y Price, 2017). La capacidad de trabajar eficazmente en diferentes niveles de complejidad es un requisito crítico en un mundo cada vez más definido por la integración de tecnologías (generalmente referidas bajo la rúbrica de inteligencia artificial), pero también en campos específicos y florecientes como la robotización (Romero, 2017) y el diseño integral.

\subsection{Reduciendo la brecha entre la educación maker y el desarrollo de competencias del siglo XXI}

Macro-diseminación de la cultura maker: promoviendo competencias del siglo XXI a través de un Ideatón. Jorge Sanabria-Z, Ann-Louise Davidson, Margarida Romero y Teresa Quintana.

Page 4 of 27 
La mayoría de los maestros están mal equipados para enseñar competencias tecnológicas avanzadas, como la programación creativa, a pesar de la necesidad de los estudiantes de adquirir un dominio computacional crítico y entrar con éxito en un mercado laboral que experimenta cambios rápidos e impredecibles. Como nunca antes, los estudiantes necesitan aprender a perseverar y a persistir (Sheridan et al., 2014); a resolver problemas mal definidos; y a trabajar en grupos de colaboración (Cucinelli et al., 2018; Davidson et al., 2017; Davidson \& Price, 2017). En las escuelas, las decisiones de integrar el movimiento maker a menudo implica la compra de kits electrónicos comerciales y paquetes para clase con recursos STEAM que no ofrecen actividades de aprendizaje que reflejen el verdadero potencial del movimiento maker o fomenten el desarrollo de competencias del siglo XXI.

Esta necesidad de desarrollo surge casi 40 años después de que Papert (1980) discutiera cómo las computadoras pueden revertir la relación enseñanza-aprendizaje, permitiendo a los niños controlar las computadoras, enseñando a las computadora cómo pensar y teorizar, de una manera sugerente a la de los epistemólogos que construyen sus propias estructuras intelectuales emulando el trabajo de Piaget en epistemología genética. En aquellos tiempos, las ideas de Papert fueron recibidas con gran escepticismo; no estaba del todo claro por qué era importante enseñar a los niños cómo programar robots y computadoras. Sin embargo, este asunto ya no es un tema de debate, y las pedagogías centradas en los estudiantes son muy deseables hoy en día.

En las aulas de clase maker, a menudo encontramos niños que utilizan kits, que van desde kits de invención MaKey MaKey y LittleBits, hasta Lego Mindstorms (Peppler et al., 2016), que se convierten en metáforas y modelos mentales de lo que es posible en esta llamada Cuarta Revolución Industrial. Estos kits tipifican el deseo por pedagogía maker en el aula. Sin embargo, pueden surgir dificultades con respecto a su implementación (Blikstein \& Worsley, 2016); por ejemplo, puede ser difícil ampliar su adopción, ya que no son accesibles para todos los presupuestos. También sabemos muy poco acerca de lo que los alumnos entienden a través del uso pedagógico de los kits, y cómo los hallazgos se transfieren a otros contextos y ultimadamente a la preparación para el mercado laboral.

Desde un punto de vista educativo, lo que los jóvenes aprenden a través de la cultura maker es fundamental para comprender tanto lo que significa ser un ciudadano del siglo XXI así como su valor como trabajadores y ciudadanos transnacionales en la era del Internet de las cosas (IoT) y la Industria 4.0. Sin embargo, el simple uso de kits STEAM o el ensamble de maker kits está lejos de ser suficiente como preparación para el futuro. Es necesario generar las condiciones adecuadas para contextualizar a los facilitadores que guían el proceso de aprendizaje en situaciones de educación maker, familiarizándolos con problemas relevantes, compartiendo metodologías maker y alentándolos a empatizar con los participantes durante sus actividades de curso o taller para lograr proyectos significativos.

Como una iniciativa para abordar la necesidad de los facilitadores en entornos formales y no formales por comprender el enfoque de la educación maker, se lanzó un primer curso-taller en Fundamentos Maker por el Comité de Cultura y Educación en Ecosistemas Maker, parte de la Red Temática Mexicana para el Desarrollo e Incorporación de Tecnología Educativa (Red LaTE), en colaboración con la Universidad de Guadalajara (UdeG). Esta introducción al uso de tecnologías de fabricación digital y metodologías 
educativas maker tuvo como objetivo sensibilizar a los maestros y facilitadores de niveles educativos básicos, medios y superiores, con respecto a la utilidad de estas herramientas para el desarrollo de competencias del siglo XXI en sus estudiantes. La experiencia de 5 días se basó en el Método de Inmersión Gradual (Sanabria, 2015), un enfoque pedagógico impulsado por tres módulos para el aprendizaje intuitivo: familiarización, co-creación y exhibición (UDGVirtual, 2018) (ver Figura 2).

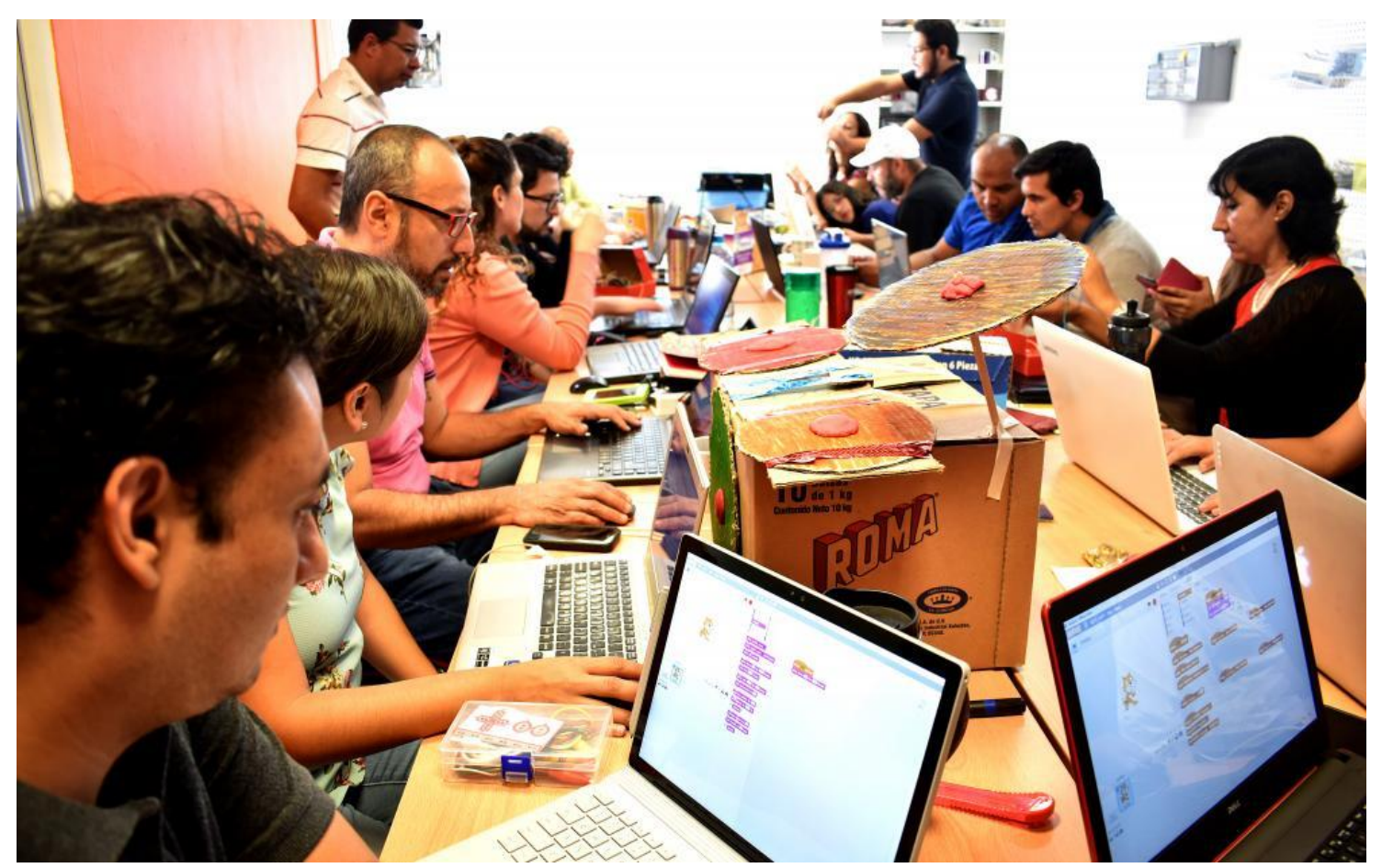

Figura 2. Primera formación docente "Fundamentos Maker", organizada en el verano de 2018 por la Red LaTE y la Universidad de Guadalajara. (Fuente: Alatorre, K., 2018)

Para continuar reduciendo esta brecha en el proceso de aprendizaje, entre la educación tradicional y la educación maker, Davidson et al. (2017) estudiaron qué conocimientos o habilidades fundamentales podrían ser necesarios para impulsar a los alumnos hacia actividades maker más relevantes socialmente. El equipo trabajó desde la premisa de que una vez que se dominan ciertos elementos fundamentales, los estudiantes pueden usarlos como una forma de moneda para resolver problemas que requieran interactuar con partes de hardware, programar, ensamblar y crear nuevas soluciones, explorando nuevas posibilidades de uso (affordances) de las tecnologías. Revelaron que una vez que adquirieron algunos conocimientos de electrónica básica, microcontroladores, robótica y creación de prototipos, y conocimientos básicos sobre la manipulación y el uso de herramientas relacionadas, incluidos sus aspectos de seguridad, los maestros y los estudiantes pueden participar en actividades más creativas porque han creado una confianza creativa en sí mismos (ver Figura 3). 


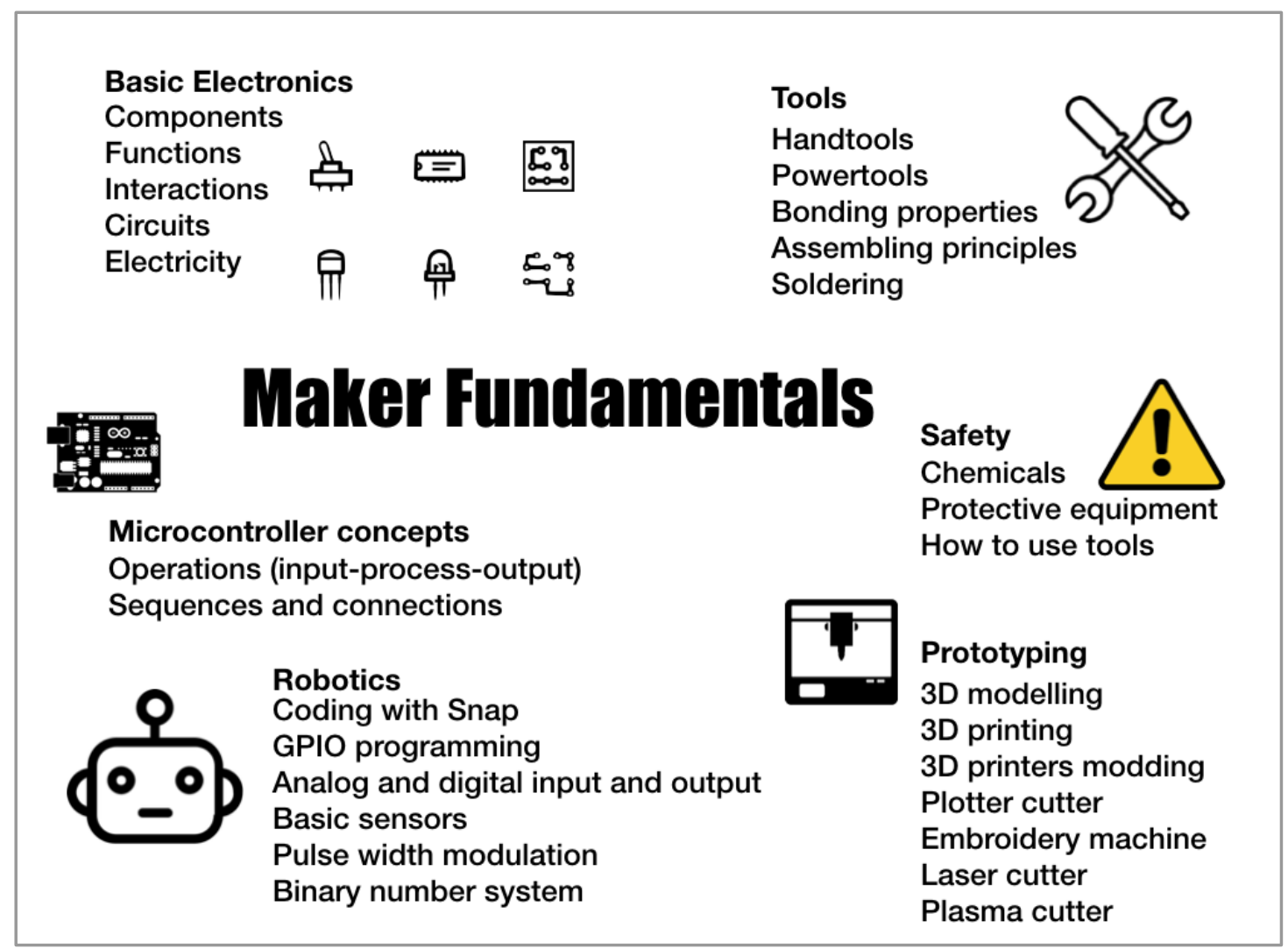

Figura 3. Fundamentos maker necesarios para participar en actividades de educación maker de nivel intermedio. (Fuente: Davidson et al., 2017)

\subsection{Competencias del siglo XXI}

Dado este contexto de proliferación de la cultura maker, debemos centrarnos no solo en el conocimiento, sino también en el desarrollo de competencias que permitan a las personas adaptarse a diferentes contextos sociales y profesionales. La cultura maker presenta un medio eficaz para el desarrollo de las competencias del siglo XXI, que responden a los desafíos educativos y socioprofesionales contemporáneos. A menudo existe una complejidad significativa en el proceso de desarrollo de prototipos en el que se sitúa un usuario, lo que implica una gran cantidad de situaciones de resolución de problemas que conducen a una reflexión crítica y creativa sobre los usos de diferentes tecnologías analógicas y digitales (Romero \& Gebera, 2012; Sanabria \& Romero, 2018), y de los materiales tradicionales y futuristas, tales como los biomateriales (Davidson \& Naffi, 2019).

El marco de competencias clave para el siglo XXI en el que se basa este artículo comprende la resolución de problemas, el pensamiento crítico, la creatividad, el pensamiento computacional y la colaboración. Cuatro de estas cinco competencias forman parte del programa de capacitación de Quebec (MEES, 2010). La competencia de pensamiento computacional es reciente y se ha integrado en varios programas de capacitación en diferentes países del mundo (ver Figura 4). 


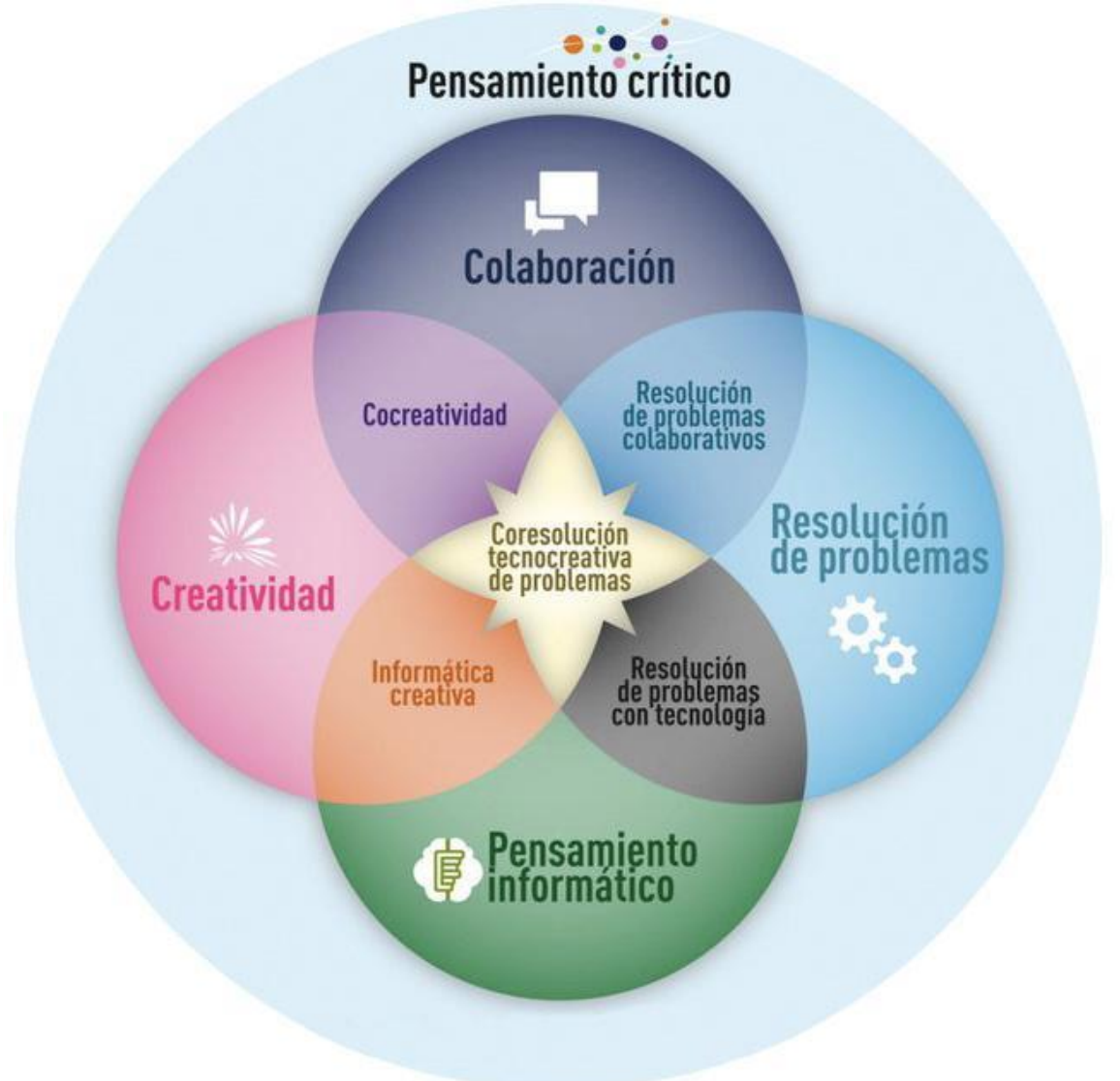

Figura 4. El Marco \#CoCreaTIC de las cinco competencias del siglo XXI. (Fuente: Romero et al., 2016).

La competencia de pensamiento crítico (representada como el fondo en la figura anterior) subsume las otras cuatro competencias, porque es necesario analizar la situación de un problema antes de definir el tipo de solución y crear el prototipo. El pensamiento computacional se considera un conjunto de estrategias mediante las cuales se resuelve un problema, con o sin tecnología, utilizando conceptos y procesos informáticos. Cuny et al. (2010) definen el pensamiento computacional como los "procesos de pensamiento involucrados en la formulación de problemas y la búsqueda de sus soluciones, de modo que las soluciones estén representadas de tal manera que puedan ser ejecutadas efectivamente por un agente que procese la información".

Desde el comienzo de la definición de la competencia computacional por Wing (2006, 2008), su relación con la competencia de resolución de problemas estuvo implícita. El acto de crear una nueva solución a través de procesos que no se conocen de antemano relaciona ambas competencias con la creatividad. Finalmente, en contextos predominantes, la mayoría de las actividades se realizan en colaboración, en grupos más o menos informales. La capacidad de trabajar en equipo, o en colaboración, es por lo tanto esencial como competencia en estos contextos, y puede transponerse a escenarios macro como los que operan en situaciones de trabajo.

\subsection{El programa tecno-creativo Ideatón como contexto de investigación}

El contexto heurístico de este artículo fue el programa Ideatón-2018 para jóvenes, parte del Festival de Innovación Epicentro, en Jalisco, México. Ideatón-2018 fue la segunda edición del programa Ideatón que se celebró por primera vez en 2017, una iniciativa de

Macro-diseminación de la cultura maker: promoviendo competencias del siglo XXI a través de un Ideatón. Jorge Sanabria-Z, Ann-Louise Davidson, Margarida Romero y Teresa Quintana.

Page 8 of 27 
la Secretaría de Innovación, Ciencia y Tecnología (SICyT) del estado de Jalisco, relanzada en colaboración con la UdeG a través de su Sistema de Universidad Virtual.

\subsubsection{Los desafíos de Jalisco y el rol de su Secretaría de Innovación, Ciencia y Tecnología (SICyT)}

La segunda década de este siglo ha sido notable por la promoción del emprendimiento en México. Entre otras iniciativas, el establecimiento de capital semilla para la inversión en nuevas empresas de alta tecnología se logró con el apoyo del banco estatal de desarrollo; el lanzamiento del Instituto Nacional del Emprendedor (INADEM), encargado de documentar y apoyar las necesidades de las pequeñas y medianas empresas; la iniciativa de colaboración entre los gobiernos de los Estados Unidos y México se consolidó mediante el desarrollo de un Consejo para el Emprendimiento y la Innovación (MUSEIC), centrado en mejorar la capacidad regional a través del fortalecimiento de los ecosistemas de alto impacto; así como el constante aumento de fondos para investigación.

Algunas de las condiciones principales para el desarrollo adecuado de un ecosistema de innovación se presentaron en 2013 durante el Foro de Innovación de Alto Nivel para Legisladores Mexicanos, organizado por el Mexico Institute, parte del Centro Internacional de Académicos Woodrow Wilson; un evento que se centró en aumentar la comprensión de los beneficios y desafíos de la innovación. Estas condiciones se pueden resumir en: generación de financiamiento sólido para investigación y desarrollo; creación de caminos hacia el financiamiento disponible; establecimiento de alianzas entre las hélices de Academia/Investigación y de Industria; alentar la formación constante de redes comerciales; considerar una visión y praxis para colaboraciones internacionales; y de particular interés, fomentar una cultura de innovación (Wood et al., 2014).

El estado de Jalisco también fue vigoroso en apoyar las iniciativas de esta década empresarial, lanzando su propia Secretaría de Innovación, Ciencia y Tecnología (SICyT) en 2013. Desde su concepción, se han implementado numerosos mecanismos y estrategias para desarrollar la economía estatal a lo largo de la alineación empresarial. Además de iniciativas tradicionales como la inversión en innovación, el fortalecimiento del sector financiero y la reforma laboral o educativa, la SICyT recibió el mandato de fomentar la cultura de innovación y emprendimiento, de acuerdo con la visión experta de los presentadores del Foro de Innovación de Alto Nivel.

\subsubsection{El Festival de Innovación Epicentro}

El Festival de Innovación Epicentro surgió en 2014 como una iniciativa anual de la Secretaría de Innovación, Ciencia y Tecnología del estado de Jalisco, con el doble objetivo de desarrollar la economía local y generar bienestar en la región, y se ha convertido en uno de los eventos más grandes de su tipo en el país, atrayendo alrededor de 50,000 participantes cada año. "Epicentro tiene como objetivo fortalecer el ecosistema de emprendimiento de alto impacto de Jalisco a través de la promoción de una cultura de innovación y facilitar el acceso al conocimiento, el desarrollo de habilidades, el intercambio de experiencias, la colaboración intersectorial y las oportunidades de vinculación, para la creación y desarrollo de innovación tecnológica con alto nivel de impacto social y cívico" (Haro, 2017). 
Adicionalmente, Epicentro se centra en los siguientes objetivos secundarios: promover los espacios públicos de aprendizaje existentes en la región, que ofrecen contenido relacionado con la capacitación de agentes de cambio y la generación de innovaciones de alto impacto; el empoderamiento de la sociedad civil para la generación de emprendimientos innovadores centrados en temas sociales prioritarios, a través de soluciones escalables, con un enfoque sostenible y respaldados por procesos cocreativos; y finalmente, lograr la participación de las cuatro hélices en el ecosistema de emprendimiento de alto impacto de manera intersectorial (Haro, 2017).

La siguiente tabla resume las actividades realizadas en el Epicentro cada año, dirigidas al mercado de jóvenes entre 25 y 35 años, con un enfoque particular en el grupo de edad entre 16 y 20 años. Los eventos a gran escala se llevan a cabo en un importante centro de eventos, mientras que los eventos menores (satélite) se distribuyen a espacios públicos en el Área Metropolitana de Guadalajara; las actividades buscan generar colaboración y trabajo colectivo entre actores de más de una hélice enfocados en problemas sociales.

Tabla 1. Resumen de actividades realizadas en el Festival de Innovación Epicentro. (Fuente: Elaboración propia basada en Haro, 2017)

\begin{tabular}{|l|l|}
\hline \multicolumn{1}{|c|}{ Actividad } & \multicolumn{1}{|c|}{ Descripción } \\
\hline Ideatón & $\begin{array}{l}\text { Competición dirigida a equipos de } \\
\text { estudiantes de bachillerato cuyo objetivo } \\
\text { es proponer soluciones a problemas } \\
\text { locales alineados con los Objetivos de } \\
\text { Desarrollo Sostenible de las Naciones } \\
\text { Unidas. }\end{array}$ \\
\hline Desarrollo de negocios & $\begin{array}{l}\text { Dinámica de desarrollo profesional } \\
\text { individual para emprendedores, basada en } \\
\text { la metodología de "speed mentoring". }\end{array}$ \\
\hline Formación & $\begin{array}{l}\text { Conferencias y talleres especializados en } \\
\text { temas de emprendimiento e innovación, } \\
\text { abiertos a diferentes niveles de } \\
\text { participantes. }\end{array}$ \\
\hline Redes y reuniones de negocios & $\begin{array}{l}\text { Espacios para networking y eventos de } \\
\text { exhibición en los que se generan redes de } \\
\text { colaboración potencial. }\end{array}$ \\
\hline Retos y dinámicas grupales & $\begin{array}{l}\text { Juegos creativos y concursos celebrados } \\
\text { durante conferencias y talleres matutinos. }\end{array}$ \\
\hline Vinculación con inversores & $\begin{array}{l}\text { Proyecto de sesiones de pitch en las que } \\
\text { emprendedores presentan ideas para ser } \\
\text { financiadas por inversores. }\end{array}$ \\
\hline $\begin{array}{l}\text { Posibilidad de que los asistentes reciban } \\
\text { reconocimiento con valor curricular, } \\
\text { otorgado por la SICyT. }\end{array}$ \\
\hline
\end{tabular}

Macro-diseminación de la cultura maker: promoviendo competencias del siglo XXI a través de un Ideatón. 


\begin{tabular}{|l|l|}
\hline $\begin{array}{l}\text { Asesoramiento para emprendedores en } \\
\text { etapas tempranas. }\end{array}$ & $\begin{array}{l}\text { Actividades, talleres, charlas y } \\
\text { conferencias enfocadas en emprendedores } \\
\text { nacientes. }\end{array}$ \\
\hline
\end{tabular}

Entre las actividades realizadas en Epicentro, destaca el programa Ideatón, dado su alcance y potencial transformador para un segmento de estudiantes a punto de lanzarse al emprendimiento.

\subsubsection{El programa Ideatón}

Alineado con los Objetivos de Desarrollo Sostenible de las Naciones Unidas (Naciones Unidas, 2015), el programa Ideatón está dirigido a estudiantes de bachillerato de entre 15 y 18 años; tiene como objetivo fomentar posibles agentes de cambio a través de la generación de soluciones innovadoras para los desafíos de nuestra sociedad. Especialmente, el Ideatón busca promover un ecosistema de emprendimiento de alto impacto en etapas tempranas y fomentar una cultura de innovación a través del acceso al conocimiento, herramientas, tutoría experta y vinculación de talentos, en línea con las tendencias mundiales en innovación y tecnología (Haro, 2018).

La estructura del Ideatón consta de cinco módulos que se llevan a cabo en el transcurso de un año: la Preselección, donde la Secretaría de Innovación, Ciencia y Tecnología hace un llamado a los estudiantes del estado de Jalisco; la Competición Inicial, donde 1,000 estudiantes seleccionados por un comité de acuerdo con criterios comunicados (incluyendo edad, equilibrio de género del equipo, competencias básicas, etc. [SEMS, 2018]) compiten en el desarrollo de soluciones como primer filtro de finalistas; el Curso de Alineación, dirigido a homogeneizar las competencias de los finalistas; el Seguimiento Tecno-creativo, centrado en el acompañamiento por expertos para el desarrollo de prototipos funcionales; y la Premiación Final donde se presentan los prototipos terminados para su evaluación por parte de los jueces, y se eligen los primeros tres lugares globales (ver Figura 5).

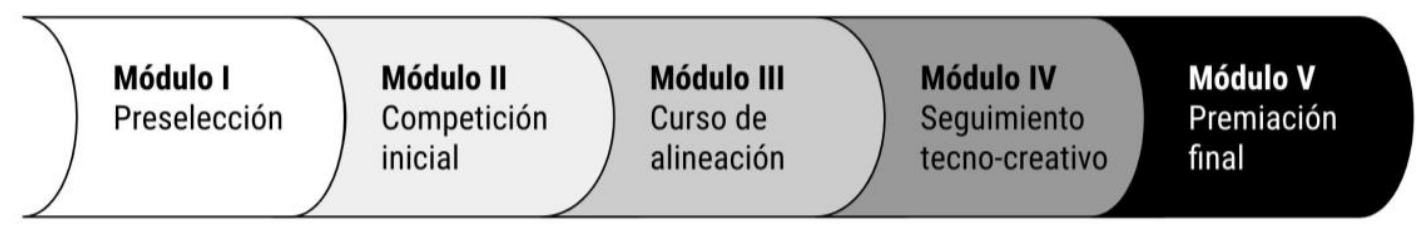

Figura 5. Los cinco módulos del programa Ideatón.

A lo largo de sus cinco módulos, el Ideatón apunta a cuatro objetivos principales: primero, desarrollar competencias del siglo XXI (colaboración, creatividad, pensamiento crítico, pensamiento computacional y resolución de problemas) a través de una competición interinstitucional; segundo, promover el prototipado rápido de alta fidelidad (e.g., interacción a través de microcontroladores, sensores y motores) (Bansemir et al., 2014), diseño y programación digital, mediante el uso de herramientas y metodologías de innovación; tercero, familiarizar a los participantes con los desafíos que enfrentan los sectores estratégicos del Estado, de conformidad con los Objetivos de Desarrollo Sostenible de las Naciones Unidas; y cuarto, generar una red de emprendedores potenciales en el ecosistema de innovación del Estado. 
Al final de los cinco módulos de su primera edición, Ideatón-2017, se analizó la logística general del programa, con base en los objetivos anteriores, en sesiones de discusión en las que participaron los coorganizadores de la Secretaría de Innovación, Ciencia y Tecnología y el investigador principal de este proyecto, Dr. Sanabria-Z, de la Universidad de Guadalajara, a fin de identificar oportunidades para mejorar el impacto tecnológico y el desarrollo de las competencias de los participantes en su próxima edición. Como resultado, se definieron los siguientes objetivos de mejora para el Ideatón2018:

1. Caracterizar el rol de los instructores y facilitadores.

2. Elevar el nivel técnico de los participantes (ampliando el alcance tecnológico de los módulos).

3. Caracterizar el rol de los participantes.

4. Aumentar el impacto en los sectores estratégicos del Estado.

5. Ampliar el contacto y seguimiento de los estudiantes.

Para alcanzar estos objetivos, con vistas al Ideatón-2018, los coorganizadores reconsideraron la logística del evento y la fortalecieron mediante la creación de un grupo de investigación internacional que se centró en el desarrollo y la evaluación de las competencias de instructores y facilitadores, así como de los participantes.

\section{Método}

El Ideatón-2018, contexto principal de este artículo, fue coorganizado por la Secretaría de Innovación, Ciencia y Tecnología (SICyT) y la Universidad de Guadalajara (UdeG). El nuevo programa replanteó la conceptualización original en términos de una colaboración que involucró la interacción entre las cuatro hélices de innovación: Gobierno y Academia/Investigación como coorganizadores, actores y evaluadores; Industria como patrocinadores, jugadores y evaluadores; y Usuarios/Sociedad como voluntarios y participantes, representados por los estudiantes y maestros involucrados. El diseño de la investigación fue concebido por el investigador principal de UdeG y complementado además por un grupo internacional formado por miembros de Concordia University (CU), en Canadá, y la Université Côte d'Azur (UCA), en Francia.

Se estructuró una investigación de métodos mixtos, con un enfoque estratégico en la indagación (Creswell \& Plano, 2007), con el fin de converger datos cualitativos y cuantitativos que, al final del proyecto, podrían contribuir a caracterizar el efecto del Ideatón como un proceso para desarrollar y evaluar las competencias del siglo XXI, con vistas a la innovación y el emprendimiento.

La investigación se construyó desde la perspectiva del pragmatismo (Creswell, 2009), basada en las siguientes premisas: el programa Ideatón, como un proceso, produce consecuencias sociales cuyo qué y cómo deben investigarse para comprender y emancipar su impacto; el evento está interrelacionado con las cuatro hélices de innovación, cuya complejidad se puede entender mejor desde varios enfoques, a través de métodos mixtos, en lugar de limitarse a un estudio cualitativo o cuantitativo solamente; y la novedad de las prácticas que ocurren durante el Ideatón requieren flexibilidad por parte de los investigadores para recopilar y analizar datos utilizando diferentes técnicas y procedimientos. 
Para fortalecer la edición Ideatón-2018, los miembros de la Secretaría de Innovación, Ciencia y Tecnología y el investigador principal de la Universidad de Guadalajara analizaron las experiencias del Ideatón-2017, acordando los elementos básicos que deberían prevalecer en términos de logística, contenido y alcance del programa; incluyendo el número de participantes, el uso de un lienzo para ideación, el número de horas por sesión, la distribución de equipos en las salas, la actividad de prototipado, los roles del instructor y del facilitador, el uso de proyectores, la entrega de premios, el curso para alinear conocimientos, el seguimiento en espacios maker (makerspaces) y el evento final de premiación.

Con base en este análisis, se identificaron una serie de requisitos logísticos y de contenido, para su implementación en la segunda edición: 1) Incluir capacitación previa de instructores y facilitadores con respecto a la logística y el uso de dispositivos electrónicos e instrumentos de evaluación; 2) Ampliar la duración del evento de tres a cuatro días, con 250 participantes por día; 3) Aumentar el número de temas del proyecto de un sector estratégico (e.g., Salud) a ocho sectores estratégicos (e.g., Manufactura, Agroindustria, Salud, etc.) por día; 4) Diseñar una plataforma digital para vincular y monitorear a los participantes antes, durante y después del evento; 5) Cambiar de prototipado rápida de baja fidelidad (e.g., cartón y plastilina) a alta fidelidad (e.g., microcontrolador, sensores y actuadores); 6) Proporcionar seguimiento a los 1,000 participantes, no sólo a los ganadores; 7) Generar un sistema de evaluación continua del proceso creativo durante los diferentes módulos del Ideatón, en lugar de evaluar únicamente el resultado final; y 8) Redefinir y diferenciar las actividades en los cinco módulos que se realizan en el transcurso de un año.

De acuerdo con estos requerimientos, el grupo UdeG-CU-UCA propuso varios criterios para el protocolo de investigación: el desempeño de los equipos participantes en el Módulo II deberá evaluarse mediante la generación de instrumentos para evaluar las competencias del siglo XXI, con base en la herramienta \#CoCreaTIC; con respecto al monitoreo de los equipos participantes, se debe diseñar e implementar una plataforma digital; en cuanto al uso de tecnologías, se debe estudiar la autopercepción de los instructores y facilitadores antes y después de la capacitación; y, por último, para la preparación y operación del Ideatón-2018, será crucial gestionar la asistencia in situ de estudiantes de UdeG, CU y UCA.

La propuesta de diseño de logística e investigación para Ideatón-2018 fue estructurada de acuerdo con los criterios y estrategias antes mencionados. El objetivo del evento fue seleccionar tres equipos ganadores en función del proceso de desarrollo de prototipos funcionales a lo largo de un año, según las temáticas de los sectores estratégicos del estado, conforme a los diferentes módulos del programa.

En el proyecto global, los métodos de investigación para la recopilación de datos involucraron tanto a los participantes como a los instructores/facilitadores, e incluyeron: un cuestionario en línea para los participantes; un lienzo de ideación para los participantes, utilizado como instrumento para recuperar las palabras clave que los equipos usaron al crear conjuntamente prototipos; un cuestionario para instructores/facilitadores; auto-evaluación de instructores/facilitadores y participantes a través de una Rueda Socrática (Chevalier \& Buckles, 2009); y la recolección de los testimonios de los ganadores a través de video. 
Ideatón-2018 fue diseñado para recibir a 1,000 estudiantes, entre 15 y 18 años, de bachilleratos públicos y privados del estado de Jalisco. El objetivo de cada equipo era desarrollar un prototipo tecnológico a través de la capacitación maker facilitada por especialistas, como solución a un reto alineado con los Objetivos de Desarrollo Sostenible de las Naciones Unidas y las megatendencias en innovación y tecnología.

Los instrumentos para la evaluación del aprendizaje y el desempeño de los estudiantes se diseñaron teniendo en cuenta la herramienta de observación \#CoCreaTIC, que enfoca en medir las competencias del siglo XXI, tal se describió en el marco teórico en la Sección 1.4 .

Durante el desarrollo y la preparación de los cinco módulos, se diseñaron varios instrumentos para su aplicación en el Ideatón, centrándose en la autopercepción y mediación de los instructores, la autopercepción de los estudiantes y el desarrollo de competencias, y el proceso creativo plasmado por los equipos en el lienzo de ideación durante el desarrollo de prototipos.

\section{Resultados}

De acuerdo con los objetivos de la investigación, se presentan a continuación los resultados de las mejoras incorporadas en los cinco módulos del Ideatón-2018.

\subsection{Mejoras incorporadas en los cinco módulos del Ideatón-2018}

El enfoque de mejora iterativa de la educación maker no sólo es un objetivo educativo del Ideatón, sino también una estrategia en sí misma que puede mejorarse año con año. Las sesiones que involucraron a miembros de la Secretaría de Innovación, Ciencia y Tecnologías y al investigador principal de la Universidad de Guadalajara ofrecieron la oportunidad de analizar oportunidades para mejorar el impacto del Ideatón-2017, y aquí comparamos los contenidos de este evento con los de la versión replanteada del Ideatón2018 .

El Módulo I, relacionado con la Preselección, mantuvo el objetivo de recibir a 1,000 estudiantes del estado de Jalisco. Este módulo se centró en una estrategia para contactar a las instituciones de nivel bachillerato, como participantes potenciales del Ideatón-2018.

Las mejoras estaban destinadas a garantizar una difusión más directa y activa de la convocatoria para reclutar estudiantes. Tanto para el Ideatón-2017 como para Ideatón2018, la selección de bachilleratos implicó realizar un análisis exploratorio (scouting) de las fortalezas de las instituciones seleccionadas en términos de ciencia, tecnología y emprendimiento, a través de una revisión de los enfoques de sus programas de estudio y el nivel de reconocimiento alcanzado por las instituciones. Para el Ideatón-2018, el alcance de la convocatoria se incrementó para abarcar más regiones del estado de Jalisco, fuera del Zona Metropolitana de Guadalajara. Con base en el análisis de potenciales graduados de bachillerato a ser convocados, se definió la agenda de reclutamiento de acuerdo con las siguientes estrategias.

Con respecto a los medios para contactar a los representantes de las escuelas de bachillerato públicas y privadas del estado de Jalisco, para el Ideatón-2017 solamente se utilizó el correo electrónico; sin embargo, para el Ideatón-2018, los representantes de las

Macro-diseminación de la cultura maker: promoviendo competencias del siglo XXI a través de un Ideatón. Jorge Sanabria-Z, Ann-Louise Davidson, Margarida Romero y Teresa Quintana.

Page 14 of 27 
escuelas fueron invitados a un desayuno informativo donde se discutieron los detalles del programa y el impacto del evento en sus instituciones. Además, si bien las invitaciones a Ideatón-2017 se realizaron a través de las instituciones, para el módulo Preselección del Ideatón-2018 se implementaron talleres de demostración presenciales con el fin de identificar los perfiles de los estudiantes más adecuados para los objetivos del evento, quienes fueron invitados directamente a registrarse. Durante las visitas a las instituciones, el evento fue promovido a través de charlas motivacionales sobre la ciudad en el año 2050, y la presentación de una línea de tiempo que ilustra las tendencias mundiales. Al final, se ofreció un taller de demostración rápida (1 hora), a través del enfoque de los "cuatro lentes de la innovación", donde los participantes propusieron estrategias para resolver problemas globales.

El Módulo II, Competición inicial, fue diseñado para servir como filtro principal para los 200 equipos participantes. Cada equipo estaba compuesto por cinco miembros que pasaron cuatro horas en la competición. A continuación se muestran tres vectores de mejora identificados para el Ideatón-2018.

La primera mejora fue la expansión de los sectores estratégicos. En el Ideatón-2017, el programa se ejecutó en 3 días, y cada día se centró en un solo sector estratégico: Día 1, Campo inteligente; Día 2, Vida saludable; y Día 3, Ciudad sostenible. Para el Ideatón2018, el programa se ejecutó en cuatro días (250 participantes por día); y no solo se amplió el programa para incluir ocho sectores estratégicos (i.e., Manufactura, Agroindustria, Salud, Movilidad, Moda y Diseño, Electrónica, Construcción y Gourmet), sino que las actividades atendieron todos los sectores cada día. La distribución específica estuvo vinculada a la consiguiente selección del espacio donde se realizaría el evento: el Palacio de Cultura y Congresos (Palcco) en Jalisco, considerado uno de los 10 teatros más grandes del mundo (ver Figura 6).

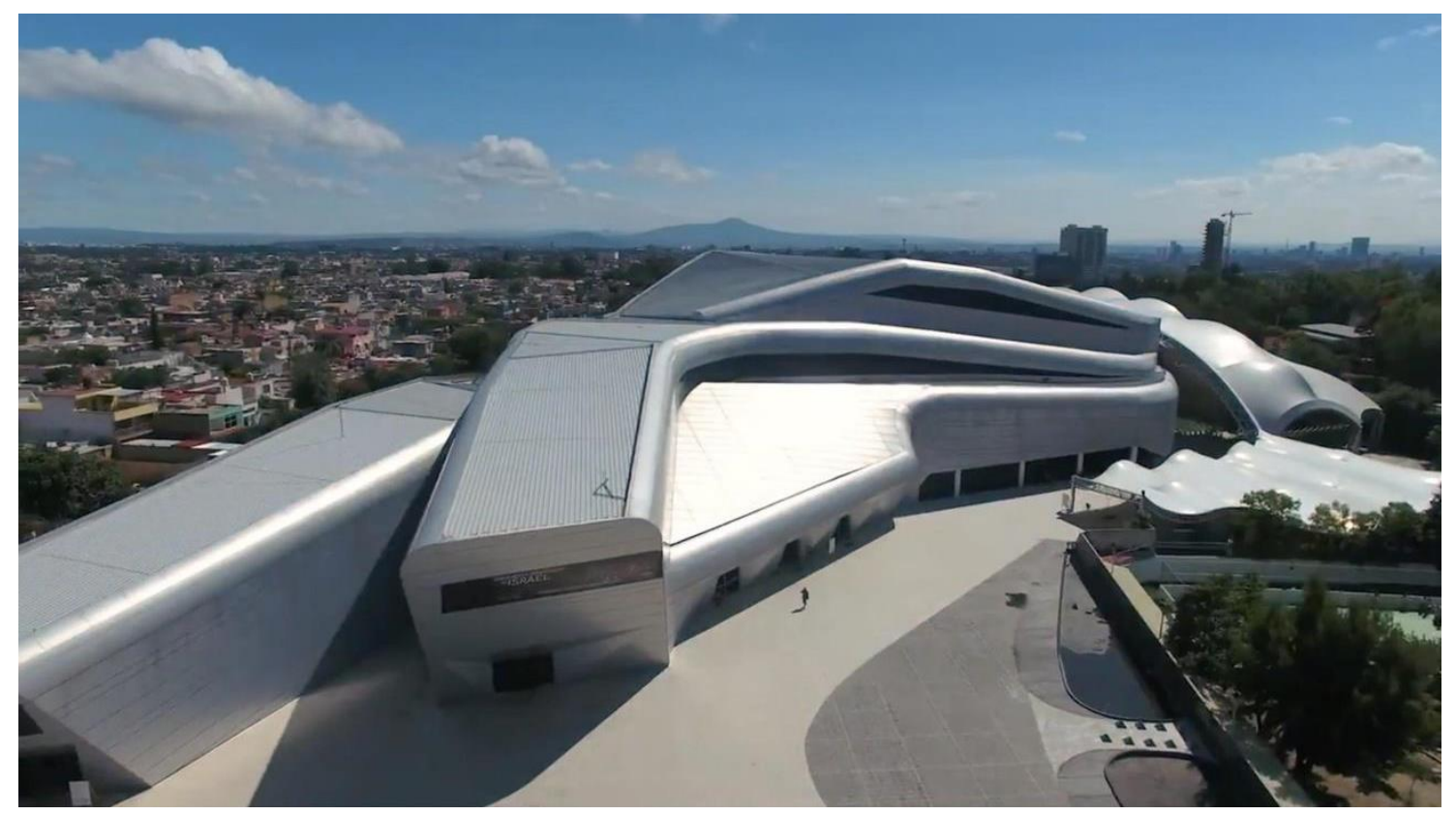

Figura 6. El Palacio de Cultura y Congresos (Palcco), en Jalisco, donde tuvo lugar el Ideatón2018. (Fuente: Epicentro Festival de Innovación, 2018a) 
En segundo lugar, se mejoró la capacitación avanzada de los facilitadores. También se realizaron mejoras en la metodología y el alcance del desarrollo de propuestas durante el evento. Basado en la experiencia del Ideatón-2017, donde se realizó un ejercicio de prototipado rápido de baja definición utilizando materiales como cartón, tijeras y pegamento, para los cuales no hubo capacitación práctica de los facilitadores e instructores, la metodología se reformuló con la intención de implementar el prototipado de alta definición. Por lo tanto, el Ideatón-2018 incluyó: la capacitación avanzada de los facilitadores e instructores sobre el procedimiento, y también sobre el uso y programación de dispositivos electrónicos; se integró el prototipado tecno-creativo, utilizando un microcontrolador programable, componentes electrónicos y una computadora, así como materiales de construcción típicos del prototipado rápido; y además se alistó un consultor experto en microcontroladores y electrónica, Robot in a can (2018), para apoyar a los equipos según fuera necesario el día del evento.

La tercera mejora se centró en la organización de las presentaciones de los equipos a lo largo del proceso de competición selectiva. En la Competición Inicial del Ideatón-2017, el objetivo era premiar a 9 equipos finalistas de los 200 participantes ( 3 equipos por día). Con este fin, se organizaron 6 salas, y en cada una se asignaron por día aproximadamente a 65 equipos de 5 estudiantes cada uno (aproximadamente 10 equipos por sala). Cada equipo presentó su proyecto final al instructor y a los facilitadores de su sala, quienes a su vez eligieron sus 3 mejores equipos, considerando el posible impacto del producto, de acuerdo con su propia experiencia y percepción, para un total de 18 equipos finalistas seleccionados por día. Estos se concentraron en un auditorio principal para resumir sus logros durante un minuto y luego responder a las preguntas de los jueces, quienes eligieron tres equipos finalistas por día en función del prototipo presentado y de su impacto.

En el caso de la Competencia Inicial en el Ideatón-2018, donde también se convocan a 200 equipos, el objetivo de premiación por día se mantuvo en 3 equipos, incrementando así la recompensa global a 12 equipos ganadores de entre los 40 finalistas, y reconociendo públicamente a estos últimos. Además de los 40 finalistas, 20 equipos destacados se reconocen también con una mención, 5 por día. (ver Figura 7).

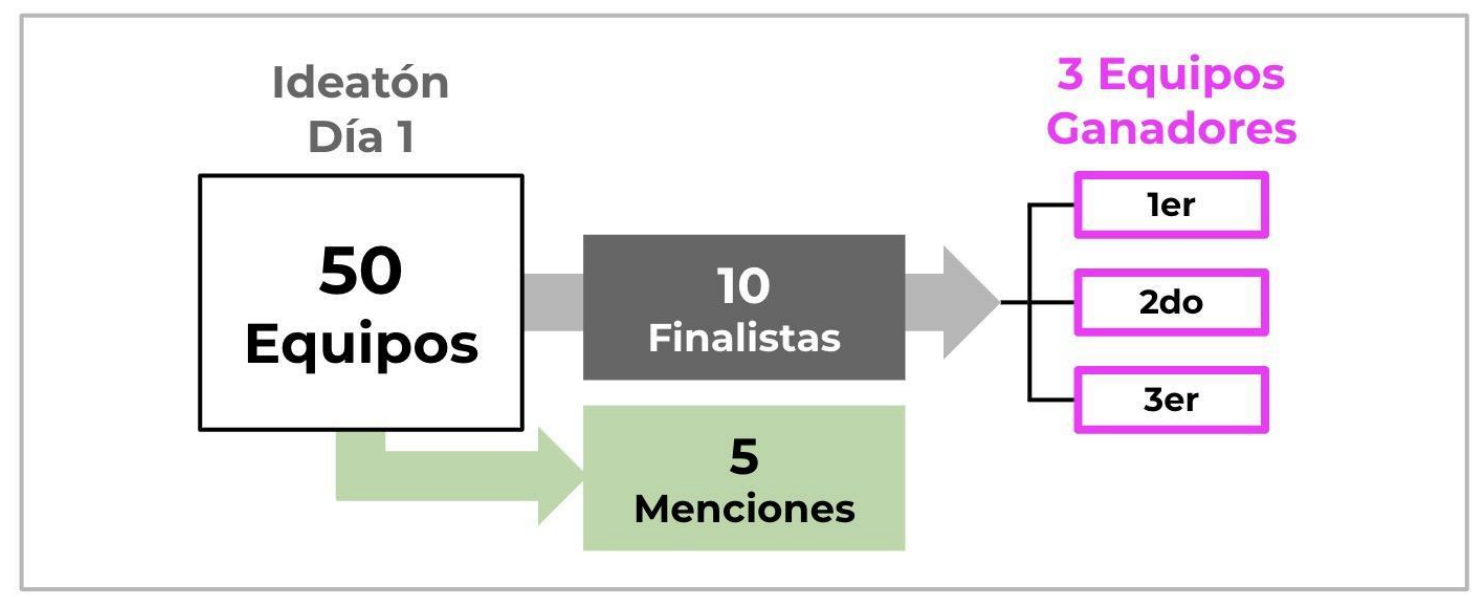

Figura 7. Proceso de selección para los tres ganadores diarios en la Competición Inicial, Módulo II del Ideatón-2018.

Cabe señalar que para permitir la continuidad del aprendizaje al término del evento, los finalistas y menciones honoríficas (i.e., 60 equipos en total) recibieron un kit tecno- 
creativo con un microcontrolador, motores y sensores, idénticos a los utilizados en el evento.

Cada día, se han de recibir 50 equipos de 5 estudiantes (si algún grupo se presenta con menos de 5 miembros, se le permite continuar el proceso pero no calificar como finalista) (ver Figura 8).

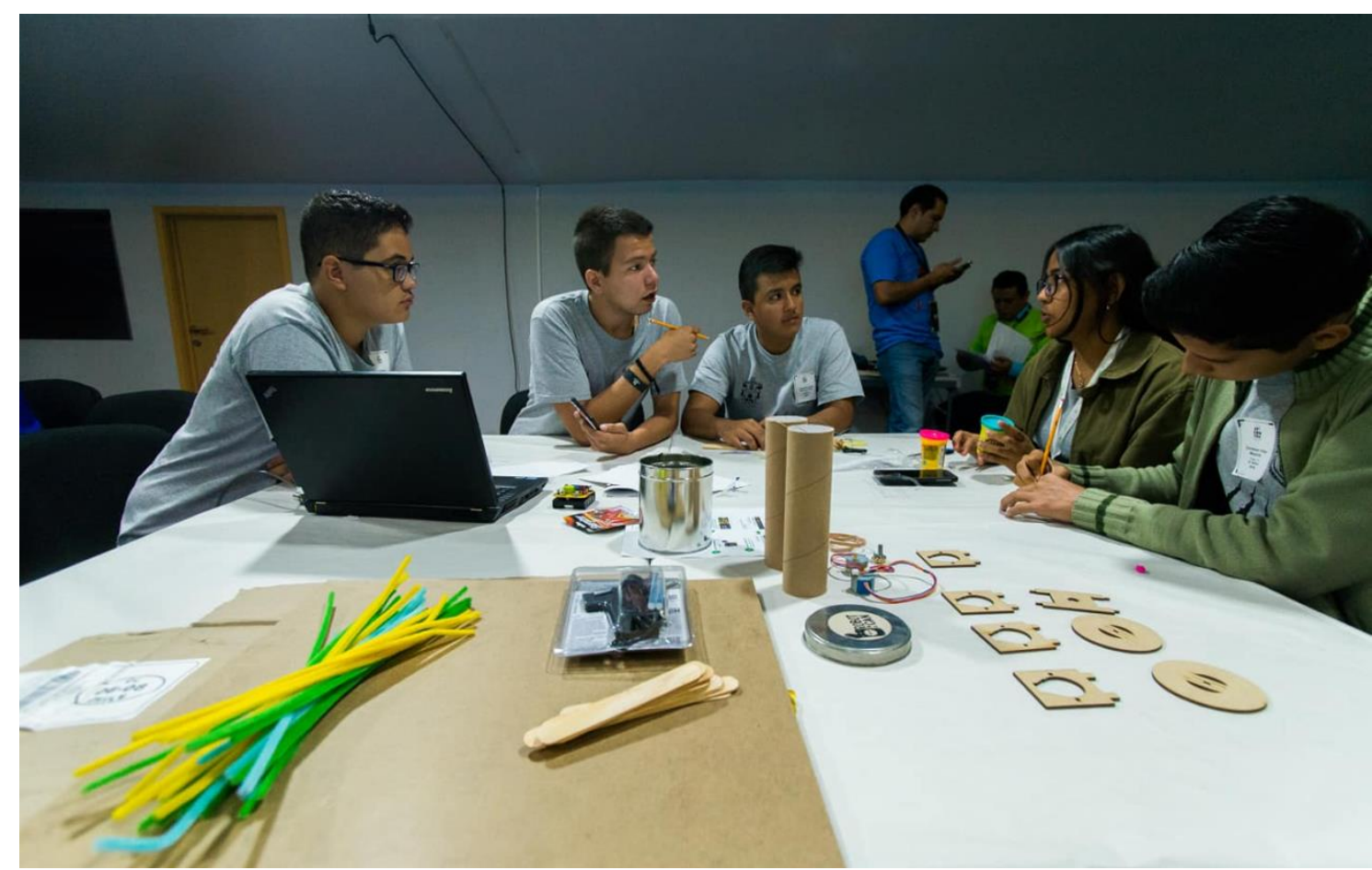

Figura 8. Equipo de cinco participantes con kit de construcción básico en el Ideatón-2018. (Fuente: Epicentro Festival de Innovación, 2018b)

Los 50 equipos se distribuyen en 10 salas (5 equipos por sala) divididas en los 8 sectores estratégicos: Manufactura, Agroindustria, Salud, Movilidad, Moda y Diseño, Electrónica, Construcción, y Gourmet. Para utilizar mejor las 10 salas, 2 de los sectores se repiten en 2 salas cada día. Por ejemplo, en el Día 1, Manufactura y Agroindustria se asignan cada uno en 2 salas, mientras que los otros 6 sectores se asignan a una sala cada uno; el día 2, Salud y Movilidad se asignan a 2 salas cada uno, y así sucesivamente. Un instructor por sala evalúa el proceso de sus 5 equipos asignados, identificando al equipo con el mejor desempeño (finalista) y al segundo mejor (mención honorífica), de acuerdo con un instrumento de evaluación basado en las competencias del siglo XXI.

La evaluación es implementada sistemáticamente por los instructores en tres momentos durante el desarrollo de los prototipos en cada sala, y concluye con una evaluación final por tres jueces en el auditorio general (ver Figura 9). 


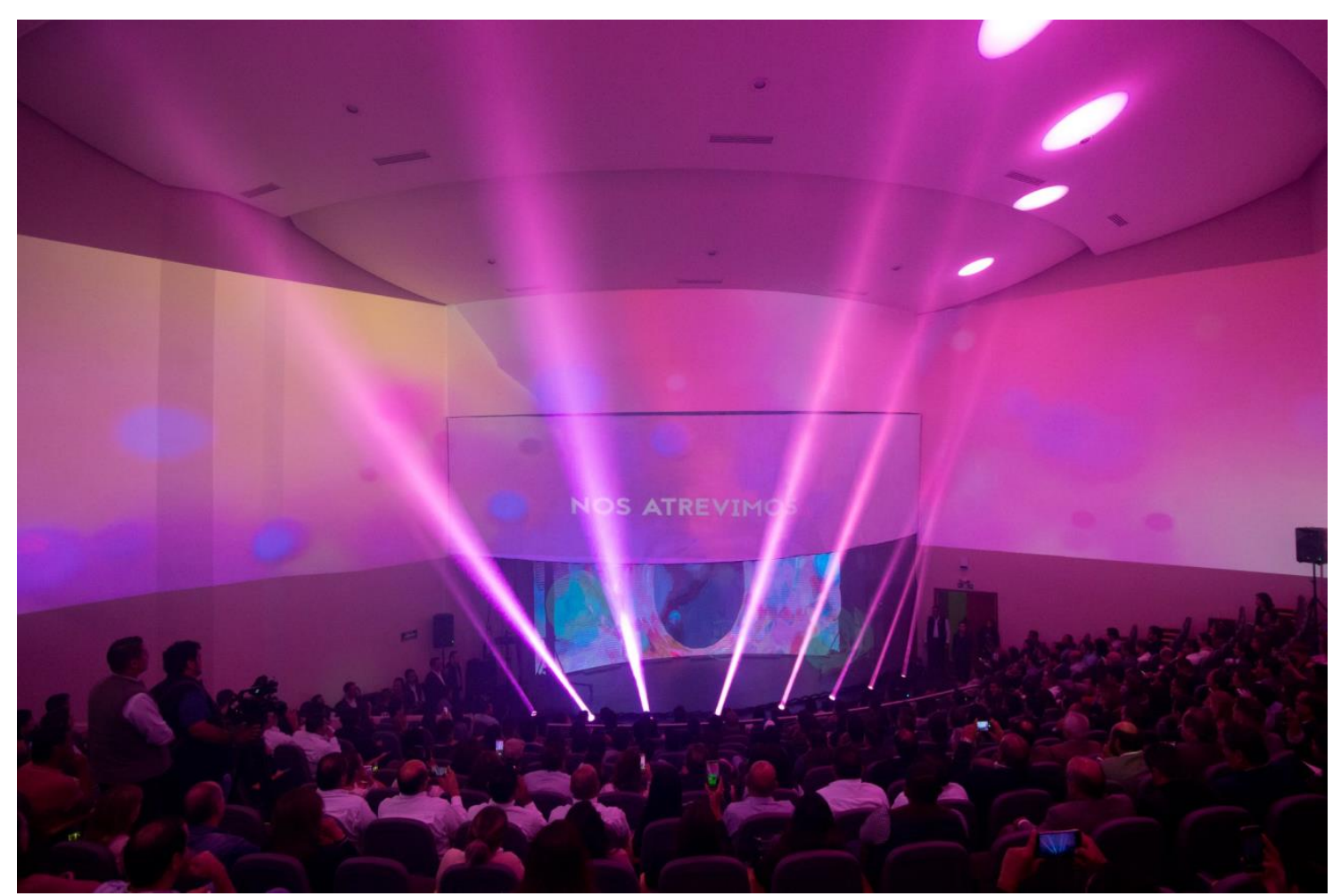

Figure 9. Auditorium where the daily winners were announced in Ideaton-2018.

(Fuente: Epicentro Festival de Innovación, 2018c)

Durante el Ideatón-2017, no hubo una herramienta para monitorear el desarrollo de las competencias del siglo XXI en relación con las tecnologías utilizadas y las soluciones propuestas. Sin embargo, para el Ideatón-2018, se concibe utilizar una plataforma digital para facilitar la interacción con los participantes a lo largo de un año, a través de sus cinco módulos (Ruby et al., 2019). Antes de la Competición Inicial, se usa para familiarizarlos con los temas de los sectores estratégicos y las tecnologías a utilizar; durante la Competición Inicial sirve como repositorio para consultas sobre los sectores estratégicos e instrucciones para el microcontrolador; para los Módulos III, IV y V, se propuso como una herramienta complementaria para los equipos participantes, enfatizando la posibilidad de desarrollar habilidades tecnológicas a través de la programación del microcontrolador y otros componentes electrónicos.

En el Módulo III, Curso de Alineación, el objetivo de motivar y capacitar a los ganadores de la Competición Inicial se mantuvo estandarizando sus competencias en términos de conocimiento técnico y visión empresarial. Las propuestas de mejora se presentan a continuación.

En el Ideatón-2017, los 9 equipos ganadores se reunieron para tomar un curso de 5 días, mientras que para el Ideatón-2018, se decidió invitar a los 60 equipos receptores de los kits tecno-creativos, distribuyendo los equipos por días para permitir concentrar una mayor atención a los ganadores. Durante el curso del Ideatón-2017, se cubrieron técnicas de diseño digital, uso de equipos de fabricación digital, comunicación asertiva y conceptos del modelo de negocio, que culminaron en una presentación por cada equipo. Para el Ideatón-2018, se propuso centrarse en el uso del microcontrolador y el diseño digital, así como en la comunicación asertiva y el modelo de negocio, también con una presentación al final. 
En el Ideatón-2017, al final de las presentaciones, empresarios y expertos ofrecieron comentarios sobre los proyectos. Para el Ideatón-2018, se agrega la generación de alianzas de acompañamiento, así como la posibilidad de recibir asesoramiento y acceso a tutoriales en línea para los 20 equipos que reciben menciones honoríficas.

Durante el Módulo IV, centrado en el Seguimiento Tecno-creativo, el programa de apoyo para los ganadores en asociación con los espacios maker se mantuvo para el Ideatón2018. Los equipos buscan desarrollar completamente sus proyectos, tanto en funcionalidad como en estética, y validarlos con futuros usuarios de acuerdo con las necesidades de estos últimos. Se propusieron las siguientes mejoras.

En el Ideatón-2017, expertos brindaron apoyo personalizado a los 9 equipos ganadores del Módulo II. Para el Ideatón-2018, además de este apoyo para los 12 equipos ganadores, se proporciona seguimiento, en modo blended (in situ y virtual), para los restantes 28 equipos finalistas y las 20 menciones honoríficas, así como tutoriales en línea en una plataforma digital (sitio web) para el global de equipos participantes. Los ganadores del Ideatón-2017 se desempeñan como "embajadores" en el Ideatón-2018, un rol ligado a la generación de cultura maker que implica participar en los diferentes módulos apoyando a los nuevos ganadores a través de la transmisión de su conocimiento y experiencia.

Para los ganadores del Ideatón-2017, se estableció una alianza con 5 espacios maker (aumentado a 12 espacios maker para el Ideatón-2018), y con empresas relacionadas con los temas de los proyectos ganadores. En esta misma línea, se propuso desarrollar llamadas para apoyo financiero, siempre que pudieran beneficiar a los 12 equipos participantes. Además, se propuso combinar la plataforma digital planteada para el Ideatón-2018, con una red social para monitorear a los 1,000 participantes.

El Módulo V, Premiación Final, mantuvo el objetivo de recompensar a los tres mejores proyectos entre los equipos ganadores de la Competición Inicial (Módulo II). Las mejoras propuestas fueron las siguientes.

El evento final del Ideatón-2017 se llevó a cabo junto con los representantes de los espacios maker que apoyaron los proyectos durante el seguimiento de los Módulos III y IV. Para el Ideatón-2018, además de los espacios de creación, se propuso agregar 12 empresas relacionadas con los temas de los proyectos de los equipos ganadores para generar potenciales alianzas.

En este evento, jueces expertos evaluaron las presentaciones y prototipos de los equipos que llegaron al evento final. Al término del Ideatón-2017, los lugares globales $1^{\circ}, 2^{\circ}$ y $3^{\circ}$ fueron recompensados, y los equipos fueron nombrados embajadores de ese Ideatón. Para el Ideatón-2018, se propuso la generación de alianzas de continuidad entre los equipos ganadores y las empresas, con el fin de registrar y desarrollar los proyectos de los equipos.

\section{Discusión}

En este artículo discutimos el contenido y la evolución de los eventos Ideatón del 2017 y 2018. A través del análisis de la experiencia del Ideatón-2017, el alcance del programa Ideatón-2018 se amplió en diferentes aspectos. La mejora del programa involucró a 
miembros de las cuatro hélices de innovación, para promover la cultura maker. El evento fue coorganizado por miembros de las hélices Gobierno y Academia/Investigación. La participación de la hélice Usuarios/Sociedad fue evidente en los maestros y estudiantes voluntarios, quienes fueron capacitados como facilitadores e instructores. Además, la integración de la hélice de la Industria se logró en forma de las propuestas de alianza entre empresarios y equipos para el desarrollo de los proyectos de los equipos.

También podemos ver un mayor impacto en términos de los sectores estratégicos abordados, de un sector por día en 2017 a ocho por día en 2018. Desde una perspectiva de investigación, el Ideatón-2018 apoyó la contribución de investigadores internacionales, lo que permitió una documentación y medición efectivas de las competencias y el desempeño de los participantes, y aumentó la relevancia social del modelo Ideatón-2018. En este sentido, el desarrollo de las competencias del siglo XXI se orientó hacia la innovación tecnológica, mediante el uso didáctico de microcontroladores programables y componentes electrónicos.

\subsection{Evaluación de competencias del siglo XXI}

La propuesta de integrar las cuatro hélices de la innovación - Gobierno + Industria, + Academia/Investigación + Usuarios/Sociedad - en el programa Ideatón-2018 es evidencia del potencial del programa para tener un impacto positivo en el ecosistema de innovación. La macro-diseminación de la cultura maker a través del desarrollo de competencias del siglo XXI, como la colaboración, la creatividad y la resolución de problemas, sienta las bases para el ecosistema de innovación y emprendimiento a mediano y largo plazo. Sin embargo, es necesario generar mecanismos e instrumentos para la medición longitudinal del desempeño de los participantes durante la cosecha del emprendimiento.

La desigualdad entre las escuelas públicas y privadas en México, la falta de laboratorios de robótica y la pérdida de oportunidades para participar en eventos de innovación o competiciones, tienen un impacto en el desarrollo de las competencias del siglo XXI del país. Esto contribuye a nuevas desigualdades en la población marginada y percibida como no calificada debido a la escuela a la que asisten, el vecindario donde viven, los estereotipos del grupo minoritario al que pertenecen o las diferencias de género. En este sentido, el objetivo es que la metodología propuesta produzca resultados justos y equilibrados para los equipos participantes de escuelas públicas y privadas, al involucrar la co-creatividad en el desarrollo de sus propuestas.

Un ecosistema de innovación se sustenta de manera compleja en su generación de una cultura de innovación. La fuerte dependencia del sistema escolar, que limita la capacidad de los jóvenes para desarrollar sus habilidades, debe superarse mediante el empoderamiento de los estudiantes que les permita percibir su potencial único. Los eventos maker y los retos de innovación ayudan a motivar a los maestros y estudiantes a desarrollar una conciencia del potencial de creación en tales eventos. Un ejemplo de la macro-diseminación de la cultura maker fue evidenciado por los propios participantes del Ideatón, quienes enriquecieron enormemente su experiencia en estos contextos, mientras continuaban desarrollando sus competencias con los colaboradores. Más específicamente, la figura del "Embajador de la Ideatón", que se otorga a los ganadores, es un mecanismo para generar cultura maker, inspirándolos a compartir sus logros y conocimientos con su entorno. 


\subsection{La dimensión de valor agregado en el concepto Ideatón}

Organizar eventos maker de gran escala es una tarea desafiante debido a la cantidad de personas involucradas, los recursos que dichos eventos consumen en términos de preparación, mano de obra, espacio, equipo y tiempo, y el hecho de que tienden a ser altamente competitivos. Como organizadores, estamos conscientes de que los participantes de eventos similares a gran escala (como los hackatones) a menudo se sienten rápidamente excluidos de ellos, en parte porque los encuentran intimidantes. También somos conscientes de las críticas comunes a tales eventos, que en general señalan que más allá de explorar tecnologías, resolver problemas de codificación, etc., a menudo hay poco valor social en tales eventos. Nos esforzamos por evitar estas posibles trampas porque nuestro objetivo es desarrollar las competencias del siglo XXI tanto en maestros como en estudiantes, para que se puedan integrar más nociones de robótica en sus respectivas escuelas; y queremos que los alumnos piensen en la innovación social mientras bricolan. La idea no es crear una invención comercializable en unos pocos días, sino crear un prototipo que tenga un valor social real y proporcionar una experiencia en la que los alumnos puedan apreciar el valor de trabajar en colaboración mientras pasan por las iteraciones de prototipado para la innovación.

Adicionalmente, en el caso del Ideatón de Jalisco, queríamos asegurarnos de que nadie saliera del evento porque se sintieron intimidados por las habilidades tecnológicas que no poseían. Con este fin, establecimos la capacitación de los instructores y facilitadores al frente del evento, con base en la suposición de que los maestros que no necesariamente sabían codificar tenían que ser entrenados para desarrollar el conocimiento fundamental necesario para guiar a los estudiantes. Si bien no pensamos en entrenarlos para que se convirtieran en programadores expertos antes del evento, proporcionamos capacitación suficiente para aumentar su nivel de confianza, o lo que Bandura (1990) llama la percepción de autoeficacia, con programación y tecno-creatividad, que son subcomponentes clave para el desarrollo de las competencias del siglo XXI.

Proporcionar suficiente preparación para los maestros (i.e., capacitar a los capacitadores) creó una estructura de apoyo para los 1,000 estudiantes participantes, que estableció una base crítica de confianza que, por ejemplo, les permitió ser guiados e influenciados por los componentes de las cuatro hélices de innovación. Primero, el Ideatón tomó en consideración las políticas gubernamentales con respecto a la educación en el siglo XXI, centradas en la construcción de la excelencia para la competitividad global y el logro de los Objetivos de Desarrollo Sostenible de las Naciones Unidas (2015). En segundo lugar, permitió a los estudiantes experimentar el contacto con componentes de los requisitos de la industria, cruciales para prepararse para el futuro del trabajo, tales como la necesidad de ser mejores solucionadores de problemas, trabajar en colaboración y pensar creativamente. En tercer lugar, alentó a todos los involucrados en los eventos a abordar las preocupaciones de los investigadores académicos que son muy críticos de integrar tecnologías por su propio bien y que exigen múltiples fuentes de evidencia antes de admitir que dicha integración tiene una influencia positiva en el aprendizaje. Cuarto, alentó a los usuarios y a la sociedad a involucrarse y participar en el desarrollo de soluciones prácticas del mundo real a los problemas que experimentan en la vida real. Finalmente, el Ideatón como concepto en evolución, contribuye a una mayor calidad de la educación para la población en general. Por ejemplo, la organización de eventos de macro-diseminación como el Ideatón, es un medio prometedor para fomentar la

Macro-diseminación de la cultura maker: promoviendo competencias del siglo XXI a través de un Ideatón. Jorge Sanabria-Z, Ann-Louise Davidson, Margarida Romero y Teresa Quintana.

Page 21 of 27 
educación maker para un gran número de estudiantes; sin embargo, como se mencionó anteriormente, tales eventos presentan desafíos logísticos en términos de apoyo institucional (e.g., transportar a la gran cantidad de estudiantes al evento) y la gestión de los facilitadores.

\section{Conclusión}

El paradigma de Innovación Abierta 2.0 (OI2), a través de la integración de las cuatro hélices, alienta a las personas a adquirir y explotar competencias que respondan efectivamente a las expectativas laborales y empresariales de las industrias contemporáneas y emergentes.

Después de haber explorado las oportunidades y los retos de la educación maker en relación con las competencias del siglo XXI, y los límites actuales de la experimentación a pequeña escala, ahora es necesario pasar de la experimentación a los eventos a gran escala. Es preciso llevar a cabo proyectos de macro-diseminación que permitan a una amplia gama de usuarios prosperar en los lugares de trabajo que enfrentan los enormes desafíos del siglo XXI.

Las valiosas actividades educativas desarrolladas en entornos maker demuestran un potencial considerable para el desarrollo de competencias del siglo XXI. Sin embargo, a pesar de este potencial, a menudo emergen experimentos limitados a pequeñas escalas que se desarrollan en esfuerzos aislados o solo alcanzan un número limitado de usuarios; por ejemplo, el EspaceLab en la biblioteca de la ciudad de Quebec, donde, a pesar de su ubicación en un contexto de biblioteca pública y abierta, el número de personas involucradas en actividades de educación para makers sigue siendo pequeño (Romero, 2016). En otros contextos, se han desarrollado espacios maker en escuelas y otros entornos en los que las actividades maker están destinadas a que toda la comunidad acceda a ellas; por ejemplo, el Fab Lab de la Facultad de Ciencias de la Université Côte d'Azur y el FabAzur en Francia (Sanabria-Z \& Romero, 2019).

El macro-evento Ideatón-2018 proporcionó a mil jóvenes acceso a los recursos y procesos del movimiento maker en educación. El uso de plataformas digitales para vincular y monitorear a los 1,000 participantes es una estrategia prometedora para generar sinergia entre ellos y alentar un interés sostenido en sus proyectos, desde compartir tutoriales hasta convocatorias y noticias relacionadas con la innovación y el emprendimiento.

Vivimos en una época de gran incertidumbre y un cambio tecnológico masivo, en el que la automatización, la robótica y la inteligencia artificial ofrecen una promesa, pero al mismo tiempo amenazan con transformar nuestras vidas y nuestros trabajos (Brynjolfsson y McAfee, 2012; Romero et al., 2018). El enfoque de cada comunidad sobre el uso de la tecnología para la co-creación de nuevas tecnologías, junto con el desarrollo de las competencias del siglo XXI, ofrece la posibilidad de empoderar a los ciudadanos para que se conviertan en miembros activos de la cultura maker, integrando las cuatro hélices de innovación y explotando estas competencias clave para prosperar en la economía creativa. 
Presentación del artículo: XX

Fecha de aprobación: 10 de octubre de 2019

Fecha de publicación: 31 de marzo de 2020

Sanabria-Z, J., Davidson, A.-L., Romero, M. \& Quintana, T. (2020) Macro-diseminación de la cultura maker: promoviendo competencias del siglo XXI a través de un Ideatón. RED. Revista de Educación a Distancia, 62. DOI: http://dx.doi.org/10.6018/red.382591

\section{Financiación}

Esta investigación ha sido apoyada por el Sistema de Universidad Virtual de la Universidad de Guadalajara, en México; la Cátedra de Investigación (Research Chair) en Cultura Maker de Concordia University (430-2016-01237), Canadá; la Université Côte d'Azur a través del ANR CreaMaker (ANR-18-CE38-0001), en Francia; la Red Temática Mexicana para el Desarrollo e Incorporación de Tecnología Educativa (Red LaTE); y ATI Tecnología Integrada.

\section{Reconocimientos}

Los autores agradecen el apoyo de la Secretaría de Innovación, Ciencia y Tecnología (SICyT); del Laboratorio de formación maker (FORMAK) del Sistema de Universidad Virtual de la Universidad de Guadalajara; del Laboratorio Education Makers del Instituto Milieux de Concordia University; del Laboratorio de Innovación Educativa Digital (LINE) y el programa SmartEdTech de la Université Côte d'Azur; y del Comité de Cultura y Educación en Ecosistemas Maker de la Red LaTE.

\section{Referencias bibliográficas}

Alatorre, K. (2018). [Photographer] UDGVirtual impulsa el movimiento maker en escuelas públicas y privadas de Jalisco. https://www.udgvirtual.udg.mx/noticia/udgvirtual-impulsa-movimiento-makeren-escuelas-publicas-privadas-jalisco

Andersson, P. (2015). Digital Fabrication and Open Concepts: An emergent paradigm of consumer electronics production. (Bachelor thesis, Umeå University). http://www.diva-portal.org/smash/get/diva2:822484/FULLTEXT02

Bandura, A. (1990). Multidimensional scales of perceived academic efficacy. Stanford, CA: Stanford University.

Bansemir B., Hannß F., Lochner B., Wojdziak J., Groh R. (2014) Experience Report: The Effectiveness of Paper Prototyping for Interactive Visualizations. In: Marcus A. (eds) Design, User Experience, and Usability. Theories, Methods, and Tools for Designing the User Experience. DUXU 2014. Lecture Notes in Computer Science, vol 8517. Springer, Cham. doi: 10.1007/978-3-319-07668-3_1

Blikstein, P. \& Worsley, M. (2016). Multimodal Learning Analytics and Education Data Mining: Using computational technologies to measure complex learning tasks. The 
Journal of Learning Analytics, 220-238.

Brynjolfsson, E., \& McAfee, A. (2012). Race against the machine: How the digital revolution is accelerating innovation, driving productivity, and irreversibly transforming employment and the economy. Brynjolfsson and McAfee.

Burker, J. (2015). Going from Bits to Atoms: Programming in Turtle Blocks JS and Personal Fabrication in Youth Maker Projects. RED. Revista de Educación a Distancia, $46,7$.

Carayannis, E., \& Campbell, D. (2009). 'Mode 3' and 'Quadruple Helix': toward a 21st century fractal innovation ecosystem. International Journal of Technology Management, 46(3/4).

http://www.inderscience.com/info/inarticle.php?artid=23374.

Chevalier, J. \& Buckles, D. (2009). SAS2: Guía para la Investigación Colaborativa y la Movilización Social. Centro Internacional de Investigaciones para el Desarrollo (IDRC): Canadá. Plaza y Valdés Editores.

Creswell, J. (2009). Research design: Qualitative, quantitative, and mixed methods approaches (3rd ed.). Thousand Oaks, CA, US: Sage Publications, Inc.

Cucinelli, G., Davidson, A. L, Romero, M., \& Matheson, T. (2018). Intergenerational learning through a participatory video game design workshop. Journal of Intergenerational Relationships, 16(1-2), 146-165.

https://doi.org/10.1080/15350770.2018.1404855

Creswell, J. \& Plano, V. (2007). Designing and conducting mixed methods research. Thousand Oaks. CA: Sage.

Cuny, J., Snyder, L., \& Wing, J. (2010). Demystifying computational thinking for noncomputer scientists. Unpublished manuscript in progress. https://www.cs.cmu.edu/link/research-notebook-computational-thinking-what-andwhy.

Curry, R. (2017). Makerspaces: A beneficial new service for academic libraries? Library Review, 66(4/5), 201-212. https://doi.org/10.1108/LR-09-2016-0081

Davidson, A.-L. \& Price, D. (2017) Does Your School Have the Maker Fever? -An Experiential Learning Approach To Developing Maker Competencies. LEARNing Landscapes, 11(1), 103-120.

http://www.learninglandscapes.ca/index.php/learnland/article/view/926

Davidson, A.-L. \& Naffi, N. (2019). Faire face à un futur incertain à travers les activités "maker". Spectre, 47(1), novembre 2019, p.11-15.

Davidson. A.-L., Romero, M., Naffi, N., Duponsel, N., Cucinelli, G., Price, D., Krsmanovic, B., Ruby, I. (2017). Les attitudes et les compétences de la culture maker. In. A. Stockless, Lebage, I., Plante. P., Actes de colloque de la CIRTA, 
UQAM, Montréal, 10-11 octobre 2017, pp.187-194.

Domingo-Coscollola, M.; Onsès-Segarra, J. y Sancho-Gil, J.M. (2018). La cultura DIY en educación primaria. Aprendizaje transdisciplinar, colaborativo y compartido en DIYLabHub. Revista de Investigación Educativa, 36(2), 491-508.DOI: http://dx.doi.org/10.6018/rie.36.2.304421

Dougherty, D., (2012). The Maker Movement. Innovations. Technology, Governance \& Globalization, (7)3, 11-14.

Epicentro Festival de Innovación (2018a, October 17). Ideatón Día 3 - Equipos ganadores [Video Screen Capture].

https://www.facebook.com/epicentrofes/videos/1148808848604964/

Ibid._(2018b, October 3). [Photograph] Resumen Día 2 - Liderazgo. https://www.facebook.com/epicentrofes/photos/a.1801014803344408/1801017876 $677434 /$ type $=3 \&$ theater.

Ibid. (2018c, October 1). [Photograph] Resumen Día 1 - Transformación. https://www.facebook.com/epicentrofes/photos/a.1799870756792146/1799875933 458295/?type=3\&theater

Fleming, L. (2015). Worlds of making: best practices for establishing a makerspace for your school. Corwin Press.

Florida, R. 2014. The Rise of the Creative Class--Revisited: Revised and Expanded. Basic Books (AZ).

Fox, S. (2014). Third Wave Do-It-Yourself (DIY): Potential for prosumption, innovation, and entrepreneurship by local populations in regions without industrial manufacturing infrastructure. Technology in Society, 39(0), 18-30. https://doi.org/10.1017/CBO9781107415324.004

Halverson, E., \& Sheridan, K. (2014). The maker movement in education. Harvard Educational Review, 84(4), 495-505.

https://doi.org/10.17763/haer.84.4.34j1g68140382063

Haug, C. (2014). Here's how we did it: The story of the EPL makerspace. Feliciter,60(1), 21-23.

Hertz, G. (2012). “Interview with Matt Ratto.” In G. Hertz (Ed.) (2012). Critical Making: Conversations. Telharmonium Press: Hollywood, CA.

Haro, C. (2017). Festival de Innovación Epicentro [Thesis]. Sistema de Universidad Virtual, Universidad de Guadalajara.

Haro, C. (2018). Festival de Innovación Epicentro. Social Innovation Journal. https://socialinnovationsjournal.org/editions/issue-7-spring-summer-2011/75disruptive-innovations/2866-festival-de-innovacion-epicentro 
Klitsie, J., Price, R. \& de Lille, C. (2018). Overcoming the Valley of Death: A Design Innovation Perspective. The 21st DMI: Academic Design Management Conference. Design Management Institute.

Markham, S., Ward, S., Aiman-Smith, L., \& Kingon, A. (2010). The Valley of Death as Context for Role Theory in Product Innovation. https://onlinelibrary.wiley.com/doi/epdf/10.1111/j.1540-5885.2010.00724.x

MEES (2010). A curriculum for the 21st century. Québec Education Program. Ministère de l'Éducation et de l'Enseignement supérieur.

http://www.education.gouv.qc.ca/fileadmin/site_web/documents/dpse/formation_je unes/54156_QEP_Chapitre01_LOW.pdf

United Nations (2015). Sustainable Development Goals. [Website]. https://www.un.org/sustainabledevelopment/

Papert, S. (1980) Mindstorms: children, computers, and powerful ideas. Basic Books, Inc.

Papert, S., \& Harel, I. (1991). Situating constructionism. Constructionism, 36, 1-11.

Peppler, K., Halverson, E., \& Kafai, Y. B. (Eds.). (2016). Makeology: Makerspaces as Learning Environments (Vol. 1). Routledge.

Robot in a can (2018, March 2). Robot in a Can [Website]. https://robotinacan.com

Romero, M. (2016). Fabrication techno-créative à l'EspaceLab junior à Sainte-Foy. Québec numérique. Available at http://www.quebecnumerique.com/espacelab/

Romero, M. (2017). L'apprentissage dans un monde complexe. In M. Romero, B. Lille, \& A. Patino (Eds.), Usages créatifs du numérique pour l'apprentissage au XXIe siècle (Vol. 1, pp. 5-14). Québec: Presses de l’Université du Québec.

Romero, M., Vallerand, V., Sanabria, J. \& Pech, G. (2016). Guía de Actividades tecnocreativas para l@s niñ@s del siglo XXI. [Archivo PDF]. Slideshare. https://es.slideshare.net/margarida.romero/gua-de-actividades-tecnocreativas-parals-nis-del-siglo-xxi

Romero, M. \& Gebera, O. (2012). Serious Games para el desarrollo de las competencias del siglo XXI. RED. Revista de Educación a Distancia, (34), 1-22.

Romero, M., Villalba-Condori, K. O., \& Patino, A. (Éd.). (2018). \#CoCreaTIC, usos creativos de las tecnologías para el aprendizaje. Universidad Católica de Santa María.

Ruby, I., Davidson, A.-L. \& Sanabria, J. (2019). What do learners and facilitators learn from an innovation challenge? Bold Educators Activate Change, ISTE 2019. June 23-26, Philadelphia, Pennsylvania.

Sanabria, J. (2015). The Gradual Immersion Method (GIM): Pedagogical Transformation

Macro-diseminación de la cultura maker: promoviendo competencias del siglo XXI a través de un Ideatón. 
into Mixed Reality. Procedia Computer Science. Volume 75, 2015, Pages 369-374.

Sanabria-Z, J., (2018). A competency-based model for Maker Lab networks. 1st Conference on Maker Cultures, Concordia University, Montreal, Canada.

Sanabria, J., \& Romero, M. (2018). Competencias del siglo XXI en proyectos cotecnocreativos. Revista Mexicana de Bachillerato a Distancia, 10(19), 10.

Sanabria-Z, J., \& Romero, M. (2019). L'écosystème techno-créatif de la Métropole Nice Côte d'Azur. Des acteurs et des tiers lieux pour le développement d'une citoyenneté créative et d'une éducation aux compétences transversales. Québec, Canada : Livres en ligne du CRIRES. Retrieved from :

https://lel.crires.ulaval.ca/oeuvre/lecosysteme-techno-creatif-de-lametropole-nicecote-dazur-des-acteurs-et-des-tiers-lieux

SEMS. (2018). Convocatoria Ideatón Segunda Edición 2018. Sistema de Educación Media Superior. Universidad de Guadalajara. http://sems.udg.mx/sites/default/files/PaginaBasica/bases_convocatoria_ideaton_2 018.pdf

Sheridan, K., Halverson, E. R., Litts, B., Brahms, L., Jacobs-Priebe, L., and Owens, T. (2014). Learning in the making: A comparative case study of three makerspaces. Harvard Educational Review, 84(4), 505-531.

Sheridan, K., \& Konopasky, A. (2016). Designing for resourcefulness. makeology: Makerspaces as learning environments. In Makeology: Makerspaces as learning environments.

UDGVirtual (2018). UDGVirtual impulsa el movimiento maker en escuelas públicas y privadas de Jalisco.

https://www.udgvirtual.udg.mx/noticia/udgvirtual-impulsa-movimiento-maker-enescuelas-publicas-privadas-jalisco

Wing, J. (2006). Computational thinking. Communications of the ACM, 49(3), 33-35.

Wing, J. (2008). Computational thinking and thinking about computing. Philosophical transactions of the royal society of London A: mathematical, physical and engineering sciences, 366(1881), 3717-3725.

Wood, D., Wilson \& García (2014). Fomentando la innovación en México: Ideas del Foro de Innovación de Alto Nivel para Creadores de Política. Woodrow Wilson International Center for Scholars. ISBN 978-1-938027-41-3 